\title{
Promoting appropriate genetic testing: the impact of a combined test review and consultative service
}

\author{
Carlos J. Suarez, MD¹, Linbo Yu, MS², Natalie Downs, MS², Helio A. Costa, PhD² and \\ David A. Stevenson, MD ${ }^{4}$
}

Purpose: Genetic test misorders can adversely affect patient care. However, little is known about the types of misorders and the overall impact of a utilization management (UM) program on curbing misorders. This study aimed to identify different types of misorders and analyze the impact of a combined test review and consultative service on reducing misorders over time.

Methods:Selected genetictests were systematically reviewed between January and December 2015 at Stanford Health Care. Misorders were categorized into five types: clerical errors, redundant testing, better alternatives, controversial, and uncategorized. Moreover, consultations were offered to help clinicians with test selection.

Results: Of the 629 molecular test orders reviewed, 13\% were classified as misorders, and $7 \%$ were modified or canceled. Controversial

\section{INTRODUCTION}

Remarkable advancements in genetic and genomic technologies and knowledge over the past decade have led to a dramatic rise in the number of disorders where genetic testing has become available for diagnosis or management (GeneTests, https:// www.genetests.org/disorders). ${ }^{1}$ This rise has simultaneously been accompanied by a progressive increase in the complexity of genetic testing. ${ }^{1}$ Conversely, the training of many healthcare providers in genetics and genomics has remained limited, resulting in providers feeling unprepared and uncomfortable when ordering and interpreting genetic tests. ${ }^{2-4}$ As a result, gaps exist between the expectations of the emerging era of genomic medicine and the actual knowledge of practicing health-care providers. Such a gap may account for the high number of inappropriate genetic test orders, referred to as genetic test misorders, that have been reported by recent studies. ${ }^{5,6}$

The potential consequences of clinical test misorders, including genetic test misorders, are numerous and severe. These include unnecessary testing, patient anxiety, failure or delays in obtaining the needed test, delayed or missed diagnoses and treatment, and financial burden to patients and institutions ${ }^{5,7-9}$ Therefore, developing strategies to curtail these pitfalls is critical to providing optimal patient care. misorders constitute the most common type (42\%); however, unlike the other misorder types, they were negligibly affected by test review. Simultaneously, 71 consults were received. With the introduction of the UM program, genetic test misorders went from $22 \%$ at baseline to $3 \%$ at the end of the year.

Conclusion: Our results show that the combined approach of test review and consultative service effectively reduced misorders over time and suggest that a UM program focused on eliminating misorders can positively influence health-care providers' behaviors.

Genet Med advance online publication 26 January 2017

Key Words: genetic testing; medical errors; reduction of misorders; types of misorders; utilization management

A few studies have shown that an active utilization management (UM) program can control the cost of genetic test misorders by performing active test review. ${ }^{5,6,10}$ However, little is known about the nature and types of these misorders, and about the overall impact of a UM intervention on each of the types over time. Likewise, few studies have investigated possible strategies to prevent misorders from occurring, which may be as important as correcting misorders after they have been placed. In an effort to overcome this limitation, a combined approach of a consultative service along with active test review was used in our intervention. The aim of this report is not only to characterize the specific types of misorders but also to detail the impact of this combined UM approach on the reduction of misorders over time.

\section{MATERIALS AND METHODS}

\section{Hospital setting}

The Stanford University Medical Center is a medical complex that includes Stanford Health Care and Stanford Children's Health and is affiliated with the Stanford University School of Medicine. In addition to outpatient clinics, Stanford Health Care includes a 613-bed adult hospital, and Stanford Children's Health includes Lucile Packard Children's Hospital, a 312-bed

${ }^{1}$ Department of Pathology, Stanford University, Stanford, California, USA; ${ }^{2}$ Stanford Health Care, Stanford, California, USA; ${ }^{3}$ Department of Genetics, Stanford University, Stanford, California, USA; ${ }^{4}$ Department of Pediatrics, Division of Medical Genetics, Stanford University, Stanford, California, USA. Correspondence: Carlos J. Suarez (cjsuarez@stanford.edu) 
pediatric hospital. The clinical laboratories are part of the Department of Pathology and serve the entire medical complex.

\section{Design of the intervention}

An institutional genetic test UM program comprising a genetic counselor, a molecular pathologist, and a medical geneticist was created in late November 2014 and housed in the Department of Pathology. Between late November and December 2014, limited retrospective and prospective data of send-out genetic test orders were reviewed, but no formal interventions were implemented. The retrospective data were composed mainly of a list of the prior year's send-out test orders along with their volumes, and were primarily used to inform the selection of the test review criteria according to an estimated workload. Once the review criteria were established, the workflow for test review was pilot-tested and fine-tuned based on feedback from the send-out processing team. The prospective data collected during this pilot period were used to assess the current state of the problem and to calibrate the misorder criteria among the team members to achieve interobserver reproducibility. The latter was accomplished by independently reviewing test orders and achieving consensus when there were classification discrepancies. Then, starting in January 2015, two activities were formally implemented: review of send-out genetic test orders that met the review criteria, and consultative services for health-care providers with questions about genetic testing. Given that the UM program constitutes a quality improvement initiative, the institutional review board determined that their review was not required.

\section{Review of genetic test orders}

Send-out genetic test orders that met the following criteria were reviewed: institutional price $\geq \$ 2,500$ until June 2015 and $\geq \$ 1,800$ from July 2015 on, the presence of multiple genetic tests ordered on the same requisition, and/or request to send the genetic test to an international laboratory. The price cutoff was initially arbitrarily chosen to capture moderate- to highcomplexity molecular tests where potential errors would have a larger medical and financial impact. After a review of the test volume and re-analysis of the workflow in June 2015, it was concluded that higher volumes of test review could be supported and hence the cost inclusion criterion was lowered. Tests that met the review criteria were manually identified by the sendout processing team and routed to the UM program for review. The review process consisted of a thorough examination of each requisition form, evaluation of the patient's medical record to verify the clinical indications for testing, and comparison of different testing options and approaches for clinical utility and cost-effectiveness. If additional information was needed, the ordering provider was contacted.

The genetic counselor performed the initial review, and cases that required further medical insight were discussed with the molecular pathologist and/or the medical geneticist. Genetic tests that would provide useful information for clinical decision making in the context of the patient's medical condition were considered to have clinical utility. ${ }^{11}$ Test orders with questionable clinical utility and/or for which a recommendation for test modification was deemed appropriate were classified as misorders. The recommendations were actively provided by either phone discussion, email follow-up, or a combination of both; however, it was ultimately the provider's decision whether to keep the original order or to modify or cancel it. To ensure consistency and reproducibility in the classification of test orders as misorders, whenever the genetic counselor was uncertain, the case was discussed with the other UM team members to reach consensus. The data associated with each case reviewed were recorded in a database that contained detailed notes about the case, the recommendation(s), if any, and the outcome.

\section{Categorization of misorders}

Misorders were categorized into five groups: clerical errors, redundant testing, alternative approaches, controversial orders, and uncategorized orders. Clerical errors meant that the provider placed the wrong test order by mistake. Redundant testing occurred when a test order provided the same or overlapping information as a concurrent or prior genetic test. Alternative approaches corresponded to tests with better alternatives available based on clinical data. Controversial orders were test orders without enough scientific data of clinical utility for the specific patient's condition. Uncategorized misorders included those that did not fit in any of the other four categories. Similar to the classification of test orders, questions about the categorization of a misorder were discussed with the UM team members to reach consensus.

\section{Genetic testing consultations}

Starting in January 2015, consultation services were offered to the Stanford clinicians to help with the process of choosing the most appropriate genetic test. The consultations were received via e-mail or by phone and reviewed by the genetic counselor along with one or both of the medical directors. The review entailed a thorough assessment of the patient's medical record and an evaluation of testing options and clinical laboratories. Once the review was completed, recommendations were provided to the consulting clinician. The data associated with each consult were entered into a database.

\section{Statistical analysis}

All statistical analyses were performed in the R programming language. Statistical significance was determined using the Student's $t$-test for comparisons between groups, and regression analysis was performed by linear regression modeling.

\section{RESULTS}

\section{Test review}

A total of 629 genetic test orders for 618 patients were reviewed from January 2015 through December 2015. Most orders were high-complexity molecular tests comprising gene sequencing panels (79\%), whole-exome sequencing (18\%), and single-gene sequencing and/or deletion/duplication analysis (3\%). Of the 
orders reviewed in 2015, 13\% were classified as misorders $(n=82)$ and over half $(54 \%, n=44)$ were either modified or cancelled; the 82 misorders were associated with 81 unique patients and came from 51 ordering providers. When broken out into the five categories of misorders, approximately half of the misorders fell into the controversial group $(42 \%, n=34)$, followed by alternative approaches $(29 \%, n=24)$, clerical errors $(17 \%$, $n=14)$, redundant testing $(10 \%, n=8)$, and uncategorized misorders $(2 \%, n=2)$ (Figure 1); examples of cases included in each category are described in Table 1. Interestingly, the test review intervention prevented all of the clerical errors and redundant misorders along with many of the alternative approaches and

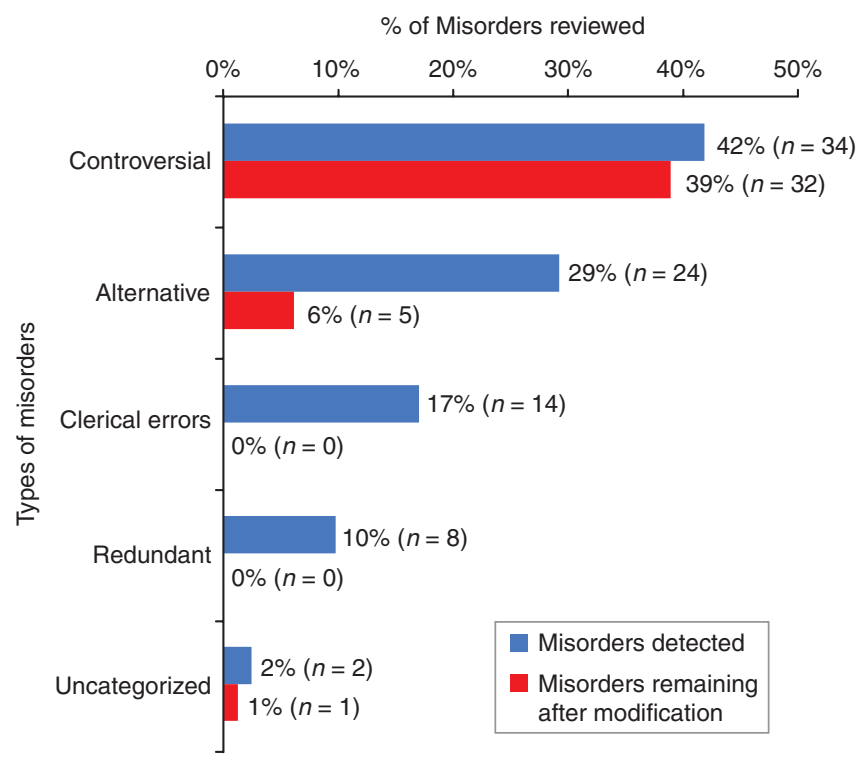

Figure 1 Effect of the test review on each type of misorder. Misorders were categorized into five types. The percentage of the total number of misorders ( $n=82$ ) represented by each type was calculated (blue bars). The percentage of misorders remaining after modifications or cancelations in each category (of the total number of misorders) was also calculated (red bars). uncategorized misorders. However, the test review intervention minimally affected the controversial misorders: only 2 of the total 34 controversial misorders were modified or canceled (Figure 1).

\section{Consultations}

Seventy-one consults were received during 2015, starting in January with 2 consults and ending with 14 and 9 consults in the last 2 months of the year, respectively (Figure 2). The reasons for these consults varied widely, from finding the most appropriate genetic panel for a condition with genetic heterogeneity, to helping a clinician decide whether genetic testing should be the next step in management. In one example, a health-care provider from a nongenetic specialty consulted the UM program to verify their planned testing strategy. The provider was considering ordering an exome-based in silico gene panel for an infant with epidermolysis bullosa. The in silico panel included 10 genes relevant for various types of epidermolysis bullosa, with $97-100 \%$ coverage, and had a turnaround time of 10-12 weeks. Upon thorough review of the patient's medical record, the patient's clinical diagnosis was most consistent with epidermolysis bullosa simplex, which is predominantly caused by mutations in the KRT5 and KRT14 genes. After comparing several different testing approaches (targeted analysis, singlegene sequencing, and next-generation sequencing panels), it was recommended to perform targeted sequencing of "hot spots" in the KRT5 and KRT14 gene first, and, if negative, then reflex to the epidermolysis bullosa in silico gene panel. The provider accepted the recommendation, and within 3 weeks the test results came back positive for a pathogenic variant in one of the hot spots. This testing approach not only shortened the time to obtain a molecular diagnosis, but also saved costs.

\section{Overall impact of the interventions}

Through the year, the occurrence of misorders trended down, from a baseline of $22 \%$ in January 2015 to $11 \%$ at the end of the

Table 1 Types of genetic test misorders

\begin{tabular}{|c|c|c|}
\hline Category & Definition & Example \\
\hline Clerical errors & Wrong test order placed by mistake & $\begin{array}{l}\text { Gene sequencing for periventricular nodular heterotopia ordered in error } \\
\text { when the intended order was flow cytometry for paroxysmal nocturnal } \\
\text { hemoglobinuria. The two conditions share the same acronym (PNH). }\end{array}$ \\
\hline $\begin{array}{l}\text { Controversial } \\
\text { misorders }\end{array}$ & $\begin{array}{l}\text { Scarce or no scientific data of the } \\
\text { test's clinical utility are available in the } \\
\text { condition it is ordered for }\end{array}$ & WES order for a patient with joint pain \\
\hline $\begin{array}{l}\text { Uncategorized } \\
\text { misorders }\end{array}$ & $\begin{array}{l}\text { Misorders that did not fit any of the } \\
\text { other four categories }\end{array}$ & $\begin{array}{l}\text { WES plus chromosomal microarray orders for a patient with } \\
\text { neuropsychiatric problems and a known diagnosis of } 22 \text { 11 deletion } \\
\text { syndrome }\end{array}$ \\
\hline
\end{tabular}

Misorders are defined as test orders with questionable clinical utility or for which a recommendation for test modification is deemed appropriate.

NGS, next-generation sequencing; WES, whole-exome sequencing. 


\section{ORIGINAL RESEARCH ARTICLE}

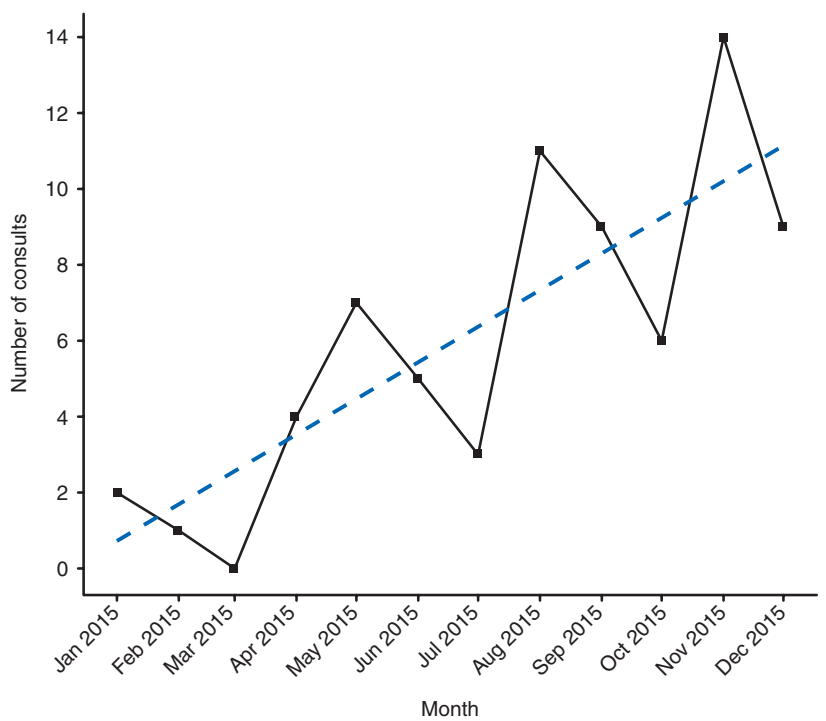

Figure 2 Change in the number of consults over time. Consults were offered from the beginning of the intervention. A statistically significant increase (dashed dark-blue line; $\mathrm{y}=0.95, \mathrm{x}=-0.24 ; r^{2}=0.61 ; P=0.0016$ ) in the number of consults over time was seen: from 2 consults in January 2015 to 14 in November 2015 and 9 in December 2015 (solid black line).

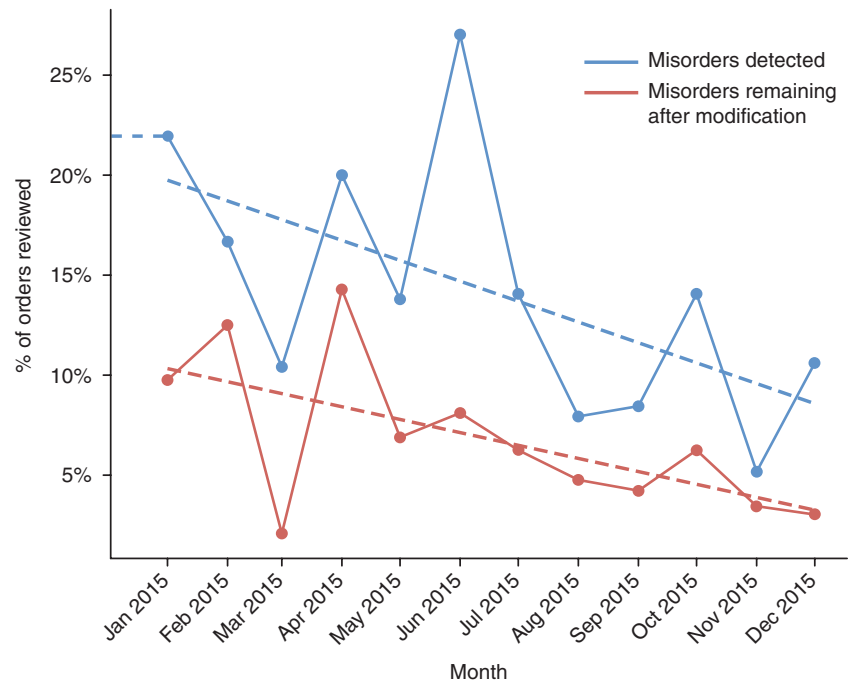

Figure 3 Changes in misorders over time. In 2015 all send-out genetic test orders that met review criteria were examined by the UM program. The percentage of misorders detected and misorders remaining after modifications were calculated for each month (of the total number of orders reviewed that month). Throughout the year, a statistically significant decrease in both the percentage of misorders detected (blue line; $y=-0.01$, $\mathrm{x}=+0.21 ; r^{2}=0.27 ; P=0.05$ ) and misorders remaining (red line; $\mathrm{y}=-0.006$, $\left.x=+0.11 ; r^{2}=0.32 ; P=0.033\right)$ was observed. The initial dotted blue line represents the predicted baseline level of misorders without interventions ( $22 \%$ misorders) according to data obtained during the first month of the intervention (January 2015).

year (Figure 3). Simultaneously, the number of modifications or cancelations of misorders increased over time, causing a decrease in the percentage of misorders remaining after the test review, from $10 \%$ of the total orders reviewed in January 2015 to 3\% in December 2015 (Figure 3). Therefore, the absolute

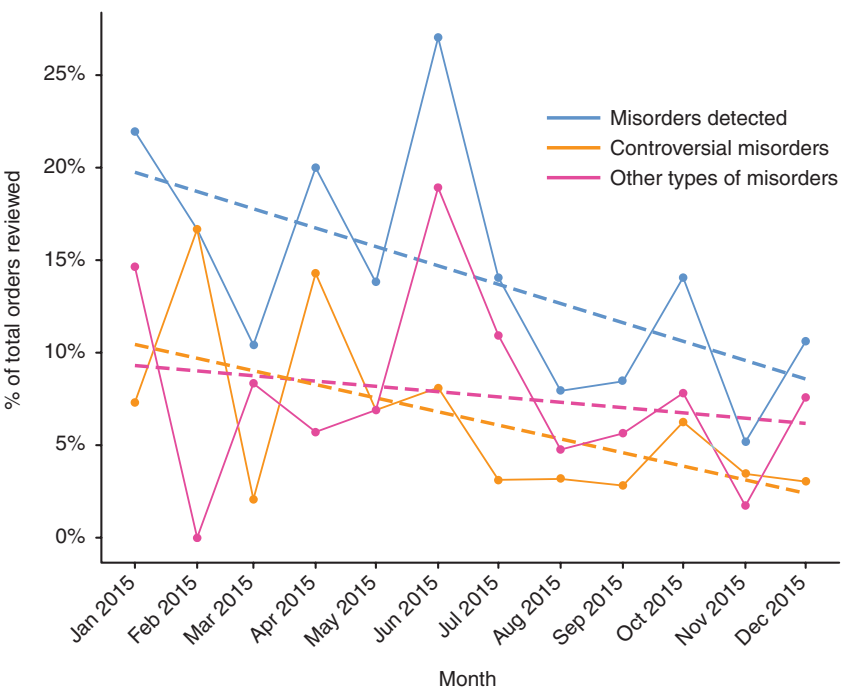

Figure 4 Changes in the occurrence of the different misorder types over time. The overall percentage of misorders detected, controversial misorders, and other types of misorders was calculated for each month (of the total number of orders reviewed that month). Through the year, while the occurrence of controversial misorders decreased (orange solid and dashed lines; $\mathrm{y}=-0.0074, \mathrm{x}=+0.11 ; r^{2}=0.25 ; P=0.057$ ), the other types of misorders combined changed minimally (pink solid and dashed lines; $\left.y=-0.0028, x=+0.096 ; r^{2}=-0.058 ; P=0.54\right)$. Most of the reduction in the overall occurrence of misorders (blue solid and dashed lines; $y=-0.01$, $\left.x=+0.21 ; r^{2}=0.27 ; P=0.05\right)$ was accounted for by the decrease in controversial misorders.

reduction of misorders in 2015 over time was 19\%, from 22\% misorders in January 2015 without interventions to 3\% remaining misorders after the test review at the final month.

Comparisons between the proportion of misorders detected and misorders remaining after modifications demonstrated a statistically significant difference between the two groups (mean difference $=7.38 \%$; 95\% confidence interval: 4.47-10.29; $P=$ 0.00016 ), suggesting that the active test review intervention led to a significant reduction in misorders. Furthermore, in addition to the reduction due to the active test review intervention, the overall occurrence of misorders also decreased over time (Figures 3 and 4), and the major contributor to that decrease was a progressive decline in controversial misorders (Figure 4). Although controversial misorders were minimally prevented by test review, their occurrence decreased at a greater rate than all other types of misorders combined (Figure 4).

Importantly, the readjustment of the cost inclusion criterion that occurred in July 2015 in order to capture tests in the $\$ 2,500$ to $\$ 1,800$ price range did not affect the aggregate results. The added price range, $\$ 1,800-\$ 2,500$, represented only $9 \%(n=$ $35)$ of the orders reviewed and $17 \%(n=7)$ of the misorders detected. Even though proportionally the price adjustment brought in a few additional misorders, it did not noticeably affect the downtrend of misorders over time (Figure 3 ). Likewise, the distribution of misorders by categories within the added price range was similar to the overall distribution, with controversial misorders composing $43 \%$ of the misorders (three 
of seven), whereas alternative approaches (two of seven) and clerical errors (two of seven) each represented approximately $29 \%$ of the misorders.

\section{DISCUSSION}

Previous studies of genetic test utilization have demonstrated the high prevalence of misorders and the financial benefit of a UM program. ${ }^{5,6,10}$ The study reported here not only confirms this high prevalence but also demonstrates the actual overall efficacy of a UM program to reduce misorders over time in a large health-care system that includes both pediatric and adult care. Our institutional UM program implemented two interventions-review of genetic tests and formal consultationsand the results suggest that it was the combination of these efforts what helped curtail all types of misorders over time.

The review of test orders is a core intervention of most UM programs, ${ }^{5}$ yet this study showed that it is not equally effective for all types of misorders. After some months of experience reviewing genetic test orders, it was discovered that misorders were not a homogeneous group, and they could be classified into five categories based primarily on what caused the misorder. The data analysis using this categorization showed that controversial misorders were the most prevalent type (42\%) and, more importantly, that the test review intervention marginally affected them. A potential explanation for the overall high prevalence of controversial misorders is that the vast majority of tests reviewed were large sequencing panels and clinical exomes. Since our knowledge about the utility of some of these tests for clinical decision making in certain conditions is still evolving, a significant portion of misorders in such groups would fall under the controversial category. Likewise, the poor effect of the test review intervention on controversial misorders could be attributed to the lack of widely accepted and precisely defined guidelines for the utilization of these complex tests, which makes it difficult to formulate solid and convincing recommendations. An American College of Medical Genetics and Genomics policy statement published in 2012 raised many important points to consider in the clinical application of genomic sequencing; it also noted that "overall analytical sensitivity is still being defined" for both whole-exome sequencing and whole-genome sequencing. ${ }^{12}$ However, more studies addressing the clinical utility of large sequencing panels and clinical exomes, followed by guidelines to direct their use, are needed to effectively address the controversial misorders.

While the test review intervenes on orders that have been already submitted, consultations constitute a proactive intervention that prevents misorders from initially occurring. Consultations enable a direct interaction with health-care providers before the orders are placed and enable the UM program to correct misorders at their origin. However, consultations are initiated by the providers, which requires their familiarity with and trust in the UM program for the consultations to occur. Our results demonstrate that throughout the year, as healthcare providers became familiar with the UM program, the number of consultations increased.
Throughout the year, the percentage of misorders remaining after the test review trended down, not only because the overall occurrence of misorders decreased but also because a higher percentage of misorders were modified upon review. The major contributor to such a positive combination was likely the reduction in the occurrence of controversial misorders. Because controversial misorders were barely affected by the test review, as their proportional representation in the monthly count of misorders declined, the proportion of modification/ cancelations increased. Likewise, because controversial misorders represented a large fraction of the monthly misorders, the progressive decrease in their occurrence had a noticeable effect on the overall occurrence of misorders. The decrease in controversial misorders may stem from the indirect effect of the intervention on the providers' behavior. Every interaction of the UM program with a provider, regardless of its motivationa test review, a consultation, or another reason-carries an educational message about the wise use of health-care resources. Such a message ultimately might lead to behavioral changes that result in outcomes such as the observed reduction in the occurrence of controversial misorders.

The scope of this study was limited in that only send-out genetic tests that met our review criteria were reviewed; these primarily included large gene panels and exome sequencing; in-house genetic tests were not included. Also, the data analyzed were only of 1 year's duration, and therefore the long-term impact of the interventions cannot be assessed yet. Another limitation is that some subjectivity might inevitably be involved in the determination and classification of misorders. However, to minimize the effect of this shortcoming, every test order was categorized by consensus among the UM members during the preintervention/training phase; then, during the intervention, all cases where the initial reviewer was uncertain about the most appropriate category were also handled in that manner. Moreover, given the subjectivity inherent in the concept of clinical utility, the definition used in the intervention was pragmatic and consistent with the recently published American College of Medical Genetics and Genomics policy statement on the subject. ${ }^{11}$ We considered that any test order that provided useful information for clinical decision making in the context of the patient's medical condition was clinically useful; likewise, the College defines clinical utility as "the likelihood that a given intervention (in this case, genetic information) will lead to an improved health outcome or to whether a test can provide information about diagnosis, treatment, management, or prevention of a disease that will be helpful to a consumer."11

In summary, this study focused on the efficacy of a genetic testing UM program to reduce misorders. Given the potential dire effects of genetic testing misorders on patient care, which extend far beyond the mere financial burden, UM programs should be seen as initiatives that offer value similar to other UM programs such as antibiotic stewardship programs. The results of this study suggest that a UM program focused on eliminating misorders can positively influence the behavior of health-care providers and decrease misorders in the course of only 1 year. 


\section{ORIGINAL RESEARCH ARTICLE}

\section{ACKNOWLEDGMENTS}

The authors thank the staff in the send-out department of the Stanford Pathology Laboratory for assistance in test identification. They also thank John Christopher, Shirley Weber, and Stanford Health Care for their support of the Utilization Management Service. In addition, the authors are grateful to the Stanford Genetic Testing Utilization Committee for their insights and guidance. H.A.C. was supported by grants from the National Institutes of Health (ClinGen 4U01HG007436-04)

\section{DISCLOSURE}

D.A.S. has received honoraria from Alexion, Guidepoint Global, Lineagen, and BMJ Best Practice. The other authors declare no conflict of interest.

\section{REFERENCES}

1. Beaudet AL. Which way for genetic-test regulation? Leave test interpretation to specialists. Nature 2010;466:816-817.

2. Mainous AG 3rd, Johnson SP, Chirina S, Baker R. Academic family physicians' perception of genetic testing and integration into practice: a CERA study. Fam Med 2013;45:257-262.

3. Nippert I, Harris HJ, Julian-Reynier C, et al. Confidence of primary care physicians in their ability to carry out basic medical genetic tasks-a European survey in five countries-Part 1. J Community Genet 2011;2:1-11.
4. Salm M, Abbate K, Appelbaum P, et al. Use of genetic tests among neurologists and psychiatrists: knowledge, attitudes, behaviors, and needs for training. J Genet Couns 2014;23:156-163.

5. Miller $C E$, Krautscheid P, Baldwin EE, et al. Genetic counselor review of genetic test orders in a reference laboratory reduces unnecessary testing. Am J Med Genet A 2014;164A:1094-1101.

6. Dickerson JA, Cole B, Conta JH, et al. Improving the value of costly genetic reference laboratory testing with active utilization management. Arch Pathol Lab Med 2014;138:110-113.

7. Miyakis S, Karamanof G, Liontos M, Mountokalakis TD. Factors contributing to inappropriate ordering of tests in an academic medical department and the effect of an educational feedback strategy. Postgrad Med J 2006;82: 823-829.

8. Qaseem A, Alguire P, Dallas P, et al. Appropriate use of screening and diagnostic tests to foster high-value, cost-conscious care. Ann Intern Med 2012;156: 147-149.

9. Zhi M, Ding EL, Theisen-Toupal J, Whelan J, Arnaout R. The landscape of inappropriate laboratory testing: a 15-year meta-analysis. PLoS One 2013;8:e78962.

10. Riley JD, Procop GW, Kottke-Marchant K, Wyllie R, Lacbawan FL. Improving Molecular Genetic Test Utilization through Order Restriction, Test Review, and Guidance. J Mol Diagn 2015;17:225-229.

11. ACMG Board of Directors. Clinical utility of genetic and genomic services: a position statement of the American College of Medical Genetics and Genomics. Genet Med 2015;17:505-507.

12. ACMG Board of Directors. Points to consider in the clinical application of genomic sequencing. Genet Med 2012;14:759-761. 